\title{
Relação entre o desempenho nos testes de esforço em esteira e de seis minutos de caminhada em pacientes com claudicação intermitente dos membros inferiores
}

\author{
Relationship between the performance of 6 minutes walk test and treadmill test in patients \\ with intermittent claudication of lower limbs
}

Mariana Abreu França', Tatiane Melo Lima², Fabio da Silva Santana³, Ozéas Lima Lins-Filho4, Gabriel Grizzo Cucato5, Crivaldo Gomes Cardoso-Júnior', Raphael Mendes Ritti-Dias 7

\begin{abstract}
Resumo
Contexto: $O$ teste de caminhada de seis minutos e o teste de esforço em esteira têm sido frequentemente utilizados para quantificação da limitação funcional dos pacientes com doença arterial periférica. Todavia, ainda não está bem estabelecido se os resultados desses testes são correlacionados.

Objetivo: Relacionar a distância total de caminhada (DTC) obtida nos testes de seis minutos e de esforço em esteira em pacientes com doença arterial periférica e sintomas de claudicação intermitente.

Métodos: A amostra foi composta por 34 pacientes (65,5 \pm 8,9 anos) de ambos os gêneros (26 homens e 8 mulheres). Os indivíduos realizaram o teste de seis minutos em corredor de 30 metros e o teste de esforço em esteira ergométrica utilizando-se protocolo específico para essa população, com intervalo de pelo menos sete dias entre os testes. Para a análise dos dados, foi utilizada a análise de correlação de Pearson.

Resultados: Houve correlação significante na DTC obtida nos testes de seis minutos e de esforço em esteira $(r=0,48, p<0,01)$. Foi observada correlação significante entre a DTC obtida nos testes nos pacientes com menor comprometimento hemodinâmico do membro $(r=0,69$; $p=0,01)$, enquanto que, nos pacientes com maior comprometimento hemodinâmico do membro, a correlação não foi significante $(r=0,03, p=0,91)$. Além disso, foi observada correlação significante entre os testes tanto nos pacientes com baixos níveis de adiposidade $(r=0,57 ; p=0,02)$ como nos pacientes com altos níveis de adiposidade $(r=0,48, p<0,05)$.

Conclusão: Os resultados deste estudo mostram que os dados obtidos do teste de seis minutos e de esforço em esteira são correlacionados, exceto em pacientes com maior comprometimento hemodinâmico do membro.
\end{abstract}

Palavras-chave: doença arterial periférica; claudicação intermitente; exercício; caminhada.

\begin{abstract} However, whether these tests are correlated remain poorly known. with peripheral artery disease and symptoms of intermittent claudication. was used for data analysis. the patients with higher limb hemodynamic commitment.

Keywords: peripheral arterial disease; intermittent claudication; exercise; walking.

Trabalho realizado na Escola Superior de Educação Física da Universidade de Pernambuco.

'Bacharel, Escola Superior de Educação Física, Universidade de Pernambuco (UPE), Recife (PE), Brasil.

${ }^{2}$ Graduanda em Escola Superior de Educação Física, Universidade de Pernambuco (UPE), Recife (PE), Brasil.

${ }^{3}$ Graduando em Educação Física na Escola Superior de Educação Física da Universidade de Pernambuco (UPE), Recife (PE), Brasil.

${ }^{4}$ Bacharel, Escola Superior de Educação Física, Universidade de Pernambuco (UPE), Recife (PE), Brasil.

${ }^{5}$ Doutorando, Escola de Educação Física e Esportes, Universidade de São Paulo (USP), São Paulo (SP), Brasil.

${ }^{6}$ Professor Doutor, Centro de Educação Física e Desportos, Universidade Estadual de Londrina (UEL), Londrina (PR), Brasil.

7 Professor Doutor, Escola Superior de Educação Física,Universidade de Pernambuco (UPE), Recife (PE), Brasil.

Fonte de financiamento: CAPES e FACEPE

Conflito de interesse: Nada a declarar.

Submetido em: 12.02.2012. Aceito em: 17.07.2012

J Vasc Bras. 2012;11(4)263-268.
\end{abstract}

Background: Six minute walk test and treadmill test have been used to evaluate the functional limitations of peripheral artery disease patients.

Objective: To analyze the relationship between the total walk distance (TWD) assessed in the six minute walk test and in the treadmill test in patients

Methods: Thirty-four patients ( $65.5 \pm 8.9$ yrs) of both genders ( 26 men 8 women) participated of the study. They performed the 6 minute walk test in a 30 meters hall and the treadmill test with a specific protocol, with an interval of at least 7 days between the tests. Pearson correlation coefficient

Results: Significant correlation was observed between TWD assessed in the six minute walk test and in the treadmill test. $(r=0.48, p<0.01)$. Significant correlation between the TWD assessed in the tests were observed in the patients with lower hemodynamic limb commitment $(r=0.69 ; p=0.01)$, while in the patients with higher hemodynamic limb commitment the correlation was not significant $(r=0.03, p=0.91)$. Furthermore, a significant correlation between TWD obtained in the tests was observed in patients with low $(r=0.57 ; p=0.02)$, and high levels of adiposity $(r=0.48, p<0.05)$.

Conclusion: The results of this study shown that walking capacity obtained with the six minute walk test and treadmill test are correlated, except in 


\section{Introdução}

O teste de esforço em esteira tem sido amplamente utilizado para avaliar a magnitude da limitação funcional de pacientes com doença arterial periférica (DAP) e sintomas de claudicação intermitente $(\mathrm{CI})^{1}$. De fato, atualmente, já existem diversos protocolos específicos para esses pacientes que permitem avaliar a severidade da limitação funcional do paciente, bem como quantificar a efetividade da terapia ${ }^{2-8}$.

Apesar disso, o teste de esforço em esteira ainda é pouco utilizado na prática clínica, o que possivelmente ocorre em razão do elevado custo do equipamento e da necessidade de profissional especializado para avaliação, bem como do tempo despendido para a realização de cada avaliação. Nesse sentido, o teste de caminhada de seis minutos, inicialmente proposto para avaliação funcional de pacientes com doença pulmonar obstrutiva crônica, tem sido utilizado em pacientes com CI, sendo considerado uma alternativa mais barata, mais rápida e com maior semelhança com as atividades de locomoção em comparação ao teste em esteira ${ }^{9}$. Além disso, o teste de seis minutos tem apresentado prognóstico equivalente aos demais protocolos de testes de esforço, sendo considerado um bom indicador de morbidade e mortalidade ${ }^{10-16}$.

Em estudo anterior, foi verificado que a distância total de caminhada (DTC) obtida nos testes de esforço em esteira foi correlacionada com a obtida no teste de seis minutos ${ }^{17}$, sugerindo que ambos os testes fornecem dados similares quanto à severidade da limitação de locomoção dos pacientes com DAP. No entanto, esse estudo se limitou a analisar um grupo heterogêneo de pacientes, de forma que ainda permanece desconhecido se a relação entre os testes ocorre em pacientes com diferentes características.

Estudos têm indicado que a capacidade de locomoção obtida no teste de esforço em esteira é influenciada por uma série de fatores, dentre os quais a hemodinâmica do membro e o seu nível de adiposidade ${ }^{7,18}$. Por outro lado, o impacto dessas variáveis na capacidade de caminhada no teste de seis minutos ainda é pouco conhecido. É possível que a influência da hemodinâmica do membro e da adiposidade corporal no teste de seis minutos seja diferente daquela observada no teste de esforço em esteira, o que poderia influenciar a relação entre os resultados do teste de seis minutos e o teste de esforço em esteira.

Assim, o objetivo do presente estudo foi analisar a relação entre a DTC obtida nos testes de seis minutos em esteira, bem como analisar a influência da hemodinâmica do membro e da adiposidade corporal na relação entre os testes.

\section{Materiais e métodos}

\section{Sujeitos}

Para identificar uma correlação significante de 0,52 , conforme observado em estudo anterior ${ }^{17}$, com um erro $a$ de 0,05 e um poder de 0,80 , é necessária uma amostra de 21 indivíduos. No sentido de garantir o poder das análises, a amostra do presente estudo foi composta por 34 indivíduos (26 homens e 8 mulheres), na faixa etária de 48 a 84 anos, recrutados em hospitais públicos e clínicas particulares de Recife-PE. Como critérios de inclusão, os pacientes deveriam apresentar índice tornozelo-braço (ITB) $\leq 0,90^{19}$, sintomas de CI há mais de seis meses da época do estudo e condições de caminhar no mínimo dois minutos com velocidade de $3,2 \mathrm{~km} / \mathrm{h}$, além de não terem sido submetidos à cirurgia de revascularização nos seis meses precedentes ao estudo. Os pacientes que conseguiam caminhar por mais de 20 minutos no teste de esforço em esteira foram excluídos.

De acordo com a Resolução no 196 de 1996, do Conselho Nacional de Saúde, todos os indivíduos foram devidamente esclarecidos sobre os objetivos e procedimentos do estudo e, posteriormente, os que concordaram em participar assinaram o termo de consentimento livre e esclarecido. Este estudo foi aprovado pelo Comitê de Ética em Pesquisa da Universidade dos autores.

\section{Variáveis clínicas}

Para a obtenção do ITB, foram mensuradas as pressões arteriais do tornozelo e do braço, por meio de doppler vascular portátil (Martec DV600, Brasil) e esfigmomanômetro de coluna de mercúrio (Unitec hospitalar, Brasil), seguindo os procedimentos descritos previamente ${ }^{20}$. O ITB foi calculado pelo quociente entre a pressão arterial do tornozelo e a pressão arterial de braço, conforme descrito anteriormente ${ }^{21}$.

A massa corporal e a estatura foram obtidas por meio de uma balança mecânica com estadiômetro acoplado (Welmy modelo 110, Brasil), com uma precisão de 0,1 quilograma e de um centímetro, respectivamente. $\mathrm{O}$ índice de massa corporal (IMC) foi calculado pelo quociente entre a massa corporal $(\mathrm{kg})$ e o quadrado da estatura $\left(\mathrm{m}^{2}\right)$.

\section{Teste de esforço em esteira}

Foi realizado teste de esforço em esteira ergométrica (Inbrasport modelo ATL) até a máxima capacidade de caminhada dos pacientes. Para tanto, foi utilizado o protocolo 
específico para indivíduos com $\mathrm{CI}^{4}$, com velocidade constante de $3,2 \mathrm{~km} / \mathrm{h}$ e incrementos de $2 \%$ de inclinação a cada dois minutos. O teste era interrompido quando os indivíduos não conseguiam mais caminhar em função da dor nos membros inferiores, momento esse identificado como DTC.

\section{Teste de caminhada de seis minutos}

O teste de seis minutos foi realizado em um corredor de 30 metros, seguindo o protocolo previamente descrito ${ }^{22}$. Para a realização do teste, os pacientes foram instruídos a completar tantas voltas quanto fossem possíveis. Foi permitido ao indivíduo parar durante o teste, caso a dor de claudicação se tornasse intolerável; porém, o cronômetro não foi interrompido durante este momento. Os pacientes que pararam de caminhar por conta da dor de claudicação foram encorajados a retornar à caminhada tão breve quanto possível. Ao final do teste, quando foi atingido o tempo de seis minutos, foi identificada a DTC. O intervalo entre o teste de seis minutos e o teste de esforço em esteira foi de pelo menos sete dias.

\section{Análise estatística}

Para a apresentação das características dos sujeitos, foi utilizada média e desvio padrão. Depois de confirmada a normalidade dos dados, foi utilizado o coeficiente de correlação de Person (r) para determinar a relação entre as DTCs obtidas nos testes de esforço em esteira e de seis minutos. Além disso, os pacientes foram estratificados de acordo com a hemodinâmica do membro (ITB $<0,55$ ou $\geq 0,55)$ e o nível de adiposidade (IMC $<26,6 \mathrm{~kg} / \mathrm{m}^{2}$ ou $\geq 26,6 \mathrm{~kg} / \mathrm{m}^{2}$ ), de acordo com a mediana do grupo, sendo que a correlação entre os desempenhos nos testes foi feita em cada um desses subgrupos. As análises foram realizadas no pacote estatístico SPSS versão 17.0. O nível de significância adotado em todas as análises foi de $\mathrm{p}<0,05$.

\section{Resultados}

As características dos pacientes incluídos no estudo estão apresentadas na Tabela 1 . A maioria dos pacientes era de idosos (73,5\%), com idade de 65,5 $\pm 8,9$ anos, com sobrepeso e com ITB moderado (variando de 0,42 a 0,76).

A Figura 1 apresenta a correlação entre as DTCs obtidas nos testes de esforço em esteira e de seis minutos. Foi observada correlação significante entre as DTCs obtidas nos testes $(r=0,523, p<0,001)$.

Nas Figuras 2 e 3, é apresentada a correlação entre as DTCs obtidas nos testes de esforço em esteira e de seis minutos, de acordo com o ITB e o IMC. Foi observada correlação significante entre a DTC obtida no teste de esforço em esteira e a obtida no teste de seis minutos nos pacientes com menor comprometimento hemodinâmico do membro (ITB $\geq 0,55 ; r=0,69 ; p=0,01$ ), enquanto que a correlação não foi significante nos pacientes com maior comprometimento hemodinâmico do membro (ITB $<0,55, \mathrm{r}=0,03, \mathrm{p}=0,91)$. Com relação ao IMC, a correlação entre os testes foi significante tanto nos pacientes com maiores níveis de adiposidade (IMC $\geq 26,6,-r=0,57 ; p=0,02$ ) como nos pacientes com menores níveis de adiposidade (IMC $<26,6, \mathrm{r}=0,48, \mathrm{p}<0,05)$.

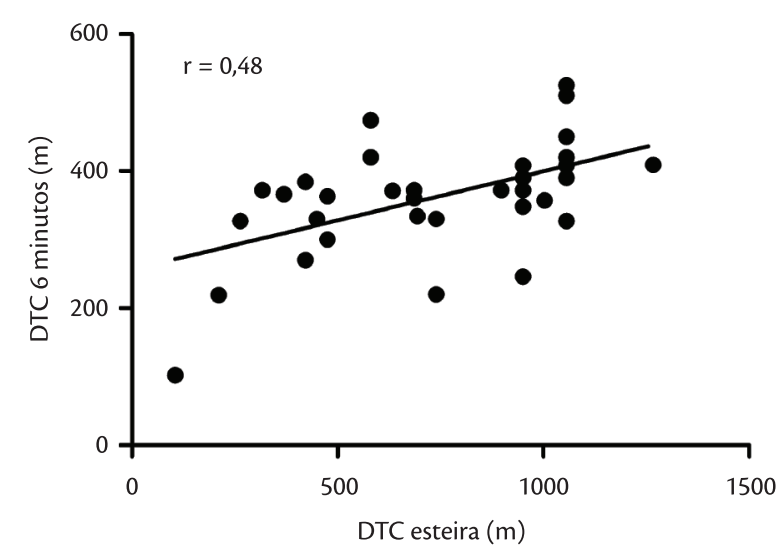

Figura 1. Correlação da distância total de caminhada (DTC) entre os testes de seis minutos e de esteira nos 34 pacientes.

Tabela 1. Características clínicas dos 34 pacientes.

\begin{tabular}{lc}
\hline Variáveis & Valores \\
\hline Idade (anos) & $65,5 \pm 8,9$ \\
Índice massa corporal $\left(\mathrm{kg} / \mathrm{m}^{2}\right)$ & $26,8 \pm 4,9$ \\
Índice tornozelo-braço & $0,63 \pm 0,12$ \\
Distância total de caminhada no teste de esforço em esteira $(\mathrm{m})$ & $904 \pm 303$ \\
Distância total de caminhada no teste de seis minutos $(\mathrm{m})$ & $361 \pm 83$ \\
\hline
\end{tabular}

Os dados são apresentados em média e desvio padrão. 
ITB $<0,55$

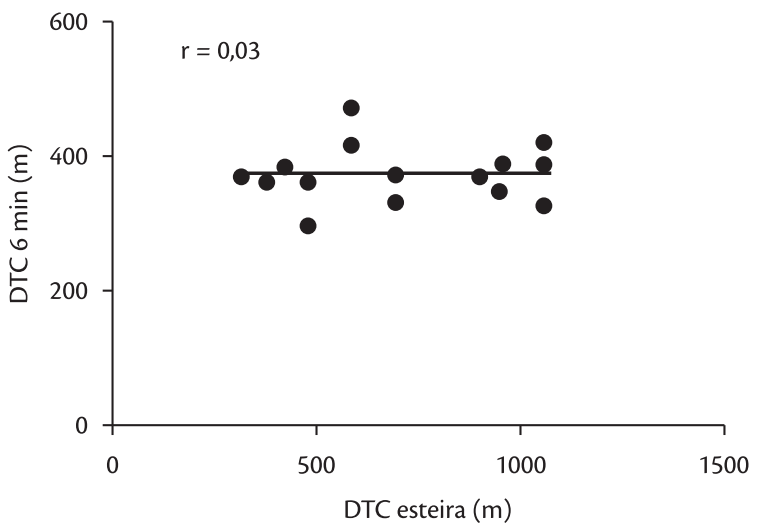

ITB $\geq 0,55$

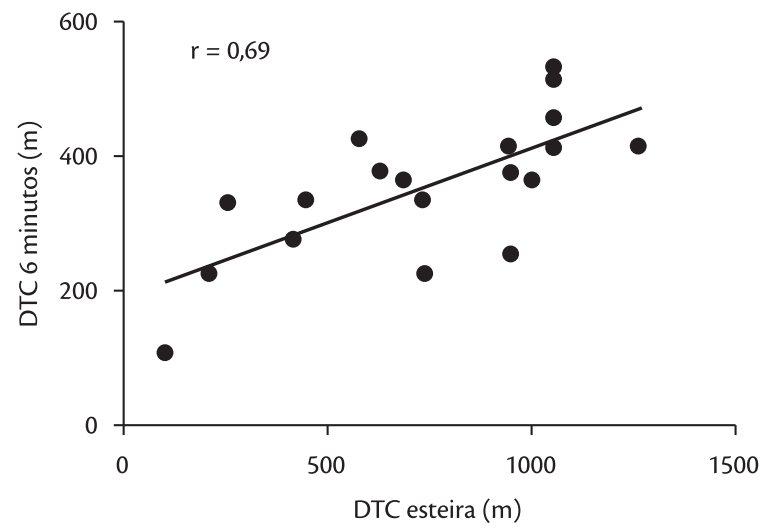

Figura 2. Correlação da distância total de caminhada (DTC) entre os testes de seis minutos e de esteira, segundo o índice tornozelo-braço (ITB).
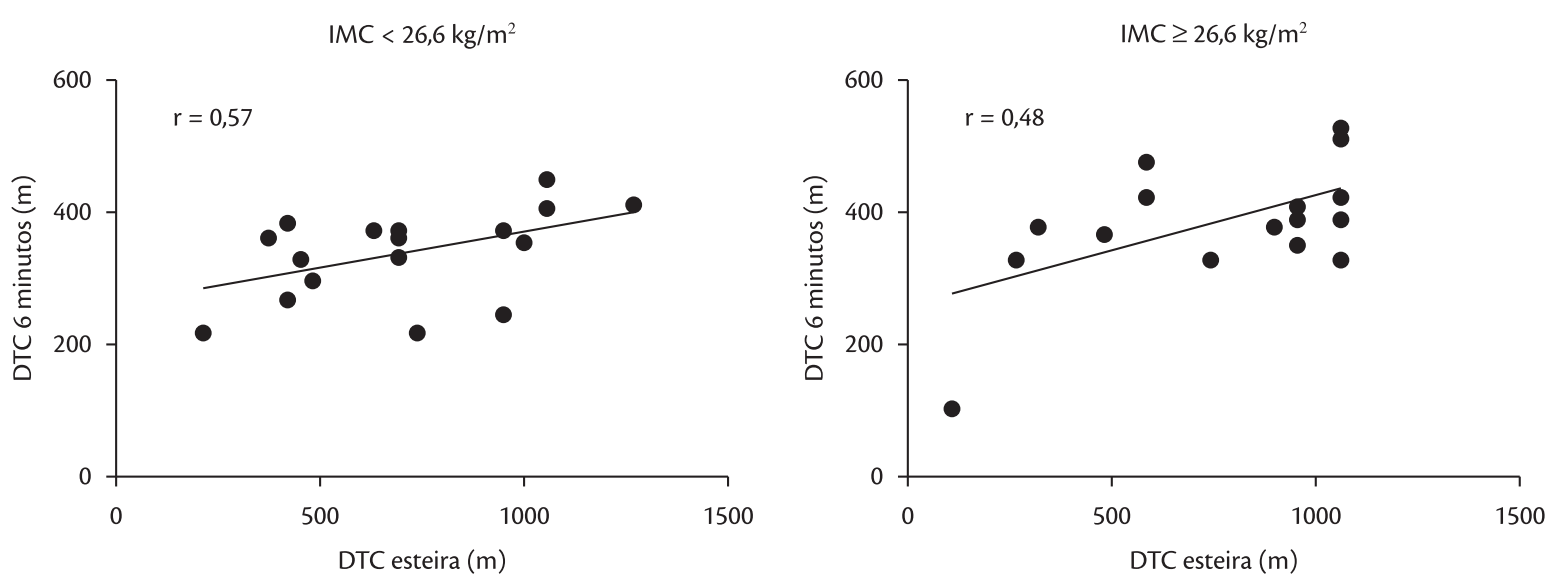

Figura 3. Correlação da distância total de caminhada (DTC) entre os testes de seis minutos e de esteira, segundo o índice de massa corporal (IMC).

\section{Discussão}

O teste de esforço em esteira e o teste de seis minutos diferem em diversos aspectos. No teste de esforço em esteira, o exercício é realizado em ergômetro, tem protocolo progressivo, a sobrecarga imposta se dá pelo aumento da inclinação da esteira e existe suporte para o apoio dos braços. Por outro lado, no teste de seis minutos, o exercício pode ser realizado num corredor, sem controle da intensidade e permite pausas para descanso durante a sua realização, caso se façam necessárias. Apesar destas diferenças, os resultados do presente estudo evidenciaram que os dados de ambos os testes apresentam relação positiva e significante. Os valores de correlação obtidos no presente estudo $(r=0,48)$ são similares aos observados em estudo anterior ${ }^{17}$ em pacientes com DAP $(r=0,52)$, indicando que ambos os testes discriminam de forma similar a capacidade funcional dos pacientes com DAP.
Com o objetivo de aprofundar o conhecimento referente à relação entre os testes de esforço em esteira e de seis minutos, foram realizadas correlações secundárias entre os respectivos testes, estratificando os pacientes de acordo com o comprometimento hemodinâmico do membro e a adiposidade corporal. Os resultados indicaram que a correlação entre os testes foi observada nos pacientes com menor comprometimento hemodinâmico do membro, enquanto que, nos pacientes com maior comprometimento, a correlação não foi significante. As causas dessa diferença na correlação não foram analisadas neste estudo; no entanto, sugere-se que isso possa ocorrer porque o teste de esforço em esteira permite maior variabilidade de resultados em comparação ao teste de seis minutos. De fato, no teste em esteira, a velocidade se mantém constante e é possível apoiar os braços durante a marcha. Com isso, o indivíduo não precisa alterar a cadência das passadas para conseguir caminhar mais. Por outro lado, no teste de seis minutos, para alcançar grandes distâncias, o paciente necessariamente precisa aumentar a 
velocidade de caminhada, o que pode precipitar o sintoma de claudicação ${ }^{23}$.

Os resultados indicaram que as correlações entre os testes de esforço em esteira e de seis minutos foram significantes tanto nos pacientes com menor adiposidade corporal como naqueles com elevada adiposidade corporal. Sabese que maiores níveis de adiposidade corporal promovem maior sobrecarga para a realização da caminhada. No entanto, em estudo anterior, foi observado que pacientes com CI obesos apresentam capacidade de caminhada similar à de pacientes eutróficos ${ }^{18}$, o que parece ocorrer pelos maiores níveis de força observados nos pacientes obesos. Dessa forma, é possível que as correlações significantes observadas nos pacientes com diferentes níveis de adiposidade corporal sejam decorrentes da maior força dos pacientes com maior adiposidade, que compensam o aumento da sobrecarga durante o esforço.

Este estudo apresenta limitações que devem ser consideradas. Embora seja possível quantificar a distância de claudicação em ambos os testes, apenas a distância total de caminhada foi utilizada como indicador de capacidade de locomoção dos pacientes. Assim, a correlação entre os testes nesse indicador não foi avaliada. Ambos os testes foram realizados uma única vez e a influência da variabilidade individual no desempenho do teste não pôde ser determinada. Por fim, este estudo analisou apenas pacientes com DAP e sintomas de CI, de forma que a extrapolação dos achados para indivíduos em outros estágios da doença não deve ser realizada.

\section{Conclusão}

Os resultados deste estudo mostram que os testes de seis minutos e de esforço em esteira são correlacionados apenas nos pacientes com menor comprometimento hemodinâmico do membro (ITB maior ou igual a 0,55 ). O nível de adiposidade corporal, avaliado pelo IMC, não interfere na correlação entre os testes estudados.

\section{Referências}

1. Norgren L, Hiatt WR, Dormandy JA, et al. Inter-society consensus for the management of peripheral arterial disease. Int Angiol. 2007;26(2):81-157. PMid:17140820.

2. Chaudhry H, Holland A, Dormandy J. Comparison of graded versus constant treadmill test protocols for quantifying intermittent claudication. Vasc Med. 1997;2(2):93-7. PMid:9546962.

3. Degischer $\mathrm{S}$, Labs KH, Aschwanden M, Tschoepl M, Jaeger KA. Reproducibility of constant-load treadmill testing with various treadmill protocols and predictability of treadmill test results in patients with intermittent claudication. J Vasc Surg. 2002;36(1):83-8. PMid:12096262.
4. Gardner AW, Skinner JS, Cantwell BW, Smith LK. Progressive vs single-stage treadmill tests for evaluation of claudication. Med Sci Sports Exerc. 1991;23(4):402-8. PMid:2056896.

5. Kirby RL. Reliability of treadmill exercise test for patients with claudication. Can J Surg. 1988;31(5):304. PMid:3416243.

6. Manfredini F, Conconi F, Malagoni AM, et al. Speed rather than distance: a novel graded treadmill test to assess claudication. Eur J Vasc Endovasc Surg. 2004;28(3):303-9. PMid:15288635. http:// dx.doi.org/10.1016/j.ejvs.2004.05.005

7. Ritti-Dias RM, Gobbo LA, Cucato GG, et al. Translation and validation of the walking impairment questionnaire in Brazilian subjects with intermittent claudication. Arq Bras Cardiol. 2009;92(2):136-49. http://dx.doi.org/10.1590/\$0066-782X2009000200011

8. Wolosker N, Ritti-Dias RM, Camara LC, Garcia YM, Jacob-Filho W, Puech-Leao P. Treadmill test is limited in elderly patients with peripheral arterial disease. Vasa. 2010;39(3):237-41. PMid:20737382.

9. ATS Committee on Proficiency Standards for Clinical Pulmonary Function Laboratories. ATS statement: guidelines for the six-minute walk test. Am J Respir Crit Care Med. 2002;166(1):111-7. PMid:12091180.

10. Cote CG, Pinto-Plata V, Kasprzyk K, Dordelly L), Celli BR. The 6-min walk distance, peak oxygen uptake, and mortality in COPD. Chest. 2007;132(6):1778-85. http://dx.doi.org/10.1378/chest.07-2050

11. Enright PL, McBurnie MA, Bittner $\mathrm{V}$, et al. The 6-min walk test: a quick measure of functional status in elderly adults. Chest. 2003;123(2):387-98. http://dx.doi.org/10.1378/chest.123.2.387

12. Ingle L, Rigby AS, Carroll S, et al. Prognostic value of the 6 min walk test and self-perceived symptom severity in older patients with chronic heart failure. Eur Heart J. 2007;28(5):560-8. http://dx.doi. org/10.1093/eurheartj/ehl527

13. Jehn $M$, Halle $M$, Schuster $T$, et al. The 6-min walk test in heart failure: is it a max or sub-maximum exercise test? Eur J Appl Physiol. 2009;107(3):317-23. PMid:19618202.

14. Martinu T, Babyak MA, O'Connell CF, et al. Baseline 6-min walk distance predicts survival in lung transplant candidates. Am J Transplant. 2008;8(7):1498-505. PMid:18510641.

15. Pinto-Plata VM, Cote C, Cabral H, Taylor J, Celli BR. The 6-min walk distance: change over time and value as a predictor of survival in severe COPD. Eur Respir J. 2004;23(1):28-33. PMid:14738227.

16. Riario Sforza GG, Incorvaia C. Mortality predictive capacity of the 6-min walk distance. Eur Respir J. 2008;32(4):1132; author reply -3. PMid:18827170

17. Montgomery PS, Gardner AW. The clinical utility of a six-minute walk test in peripheral arterial occlusive disease patients. I Am Geriatr Soc. 1998;46(6):706-11. PMid:9625185.

18. Dias RM, Forjaz CL, Cucato GG, et al. Obesity decreases time to claudication and delays post-exercise hemodynamic recovery in elderly peripheral arterial disease patients. Gerontology. 2009;55(1):21-6. PMid:18784409.

19. Management of peripheral arterial disease (PAD). TransAtlantic Inter-Society Consensus (TASC). Section B: intermittent claudication. Eur I Vasc Endovasc Surg. 2000;19 Suppl A:S47-114. PMid:10957905.

20. Wolosker N, Rosoky RA, Nakano L, Basyches M, PuechLeao P. Predictive value of the ankle-brachial index in the 
evaluation of intermittent claudication. Rev Hosp Clin Fac Med Sao Paulo. 2000;55(2):61-4. http://dx.doi.org/10.1590/ S0041-87812000000200005

21. Gardner AW, Montgomery PS. Comparison of three blood pressure methods used for determining ankle/brachial index in patients with intermittent claudication. Angiology. 1998;49(9):723-8. PMid:9756423.

22. Fontaine R, Kim M, Kieny R. [Surgical treatment of peripheral circulation disorders]. Helv Chir Acta. 1954;21(5-6):499-533. PMid:14366554.

23. Gardner AW, Montgomery PS, Scott KJ, Afaq A, Blevins SM. Patterns of ambulatory activity in subjects with and without intermittent claudication. J Vasc Surg. 2007;46(6):1208-14. http://dx.doi. org/10.1016/j.jvs.2007.07.038
Corespondência

Raphael Mendes Ritti Dias

Escola Superior de Educação Física - Universidade de Pernambuco (UPE) Rua Arnóbio Marques, 310, CEP 50100-130, Santo Amaro, Recife (PE), Brasil

E-mail: raphaelritti@gmail.com

Contribuições dos autores Concepção e desenho do estudo: RMR, GGC Análise e interpretação dos dados: CGC Coleta de dados: MAF, TML, FSS, OLL Redação do artigo: MAF, TML, FSS, OLL Revisão crítica do texto: RMR, GGC, CGC Aprovação final do artigo*: RMR, GGC, CGC, MAF, TML, FSS, OLL Análise estatística: CGC

Responsabilidade geral pelo estudo: RMR *Todos os autores leram e aprovaram a versão final submetida do J Vasc Bras. 